Algebraic 85 Geometric Topology

Volume 2 (2002) 897-919

Published: 20 October 2002

ATG

\title{
Maximal index automorphisms of free groups with no attracting fixed points on the boundary are Dehn twists
}

\author{
Armando Martino
}

\begin{abstract}
In this paper we define a quantity called the rank of an outer automorphism of a free group which is the same as the index introduced in [7] without the count of fixed points on the boundary. We proceed to analyze outer automorphisms of maximal rank and obtain results analogous to those in [4]. We also deduce that all such outer automorphisms can be represented by Dehn twists, thus proving the converse to a result in [3], and indicate a solution to the conjugacy problem when such automorphisms are given in terms of images of a basis, thus providing a moderate extension to the main theorem of [3] by somewhat different methods.
\end{abstract}

AMS Classification 20E05, 20E36

Keywords Free group, automorphism

\section{Introduction}

The celebrated result of [2] showed that for any automorphism of a finitely generated free group the rank of its fixed subgroup is at most that of the rank of the ambient free group. In [5] a detailed analysis and description was obtained for those automorphisms whose fixed subgroup has the largest possible rank maximal rank automorphisms.

The paper of [3] introduced a class of automorphisms called Dehn Twists (defined below) and showed that these have maximal index with no attracting fixed points on the boundary. In that work, the conjugacy problem for Dehn Twists is also solved and it is shown, by using the results of [5], that a maximal rank automorphism can be represented by a Dehn Twist.

In this paper we define a notion of rank for outer automorphisms which generalises the notion of rank of the fixed subgroup. (In fact this notion of rank is implicit in [2].) Alternatively, this rank can be thought of as the index of 
an outer automorphism, as described in [7], but with the change that the fixed points on the boundary are not counted.

We then proceed to generalise the results of [5] to the class of maximal rank outer automorphisms and obtain a normal form similar to the one obtained there. Moreover, we show that any such outer automorphism can be realised as a Dehn Twist. Thus the class of Dehn Twists and that of maximal rank outer automorphisms coincide.

In [9] the normal form of [5] is used to provide a solution to the conjugacy problem for maximal rank automorphisms. We also observe that since the normal form we obtain is so similar to that in [5], it is possible to use the same proof to provide a solution to the conjugacy problem for Dehn Twists by entirely different means to those of [3]. Moreover, this solution would take as input data a Dehn Twist described purely in terms of its action on a basis rather than by graph of groups data as required in [3] hence giving an extension to that result.

\section{Preliminaries}

\subsection{Outer automorphisms and index}

Throughout $F_{n}$ shall denote the free group of rank $n$. Here the rank is the minimal number of generators and is the same as the number of free generators. The rank of a subgroup, $H$, of $F_{n}$ is the least cardinality of the generating sets of the subgroup and is denoted $r(H)$.

$\operatorname{Aut}\left(F_{n}\right)$ is the group of automorphisms of $F_{n}$. $\operatorname{Inn}\left(F_{n}\right)$ will be the subgroup of inner automorphisms and $\operatorname{Out}\left(F_{n}\right)=\operatorname{Aut}\left(F_{n}\right) / \operatorname{Inn}\left(F_{n}\right)$ the group of outer automorphisms of $F_{n}$. We use the notation $\gamma_{g}$ to denote conjugation by $g$. Thus $w \gamma_{g}=g^{-1} w g$ for all $w \in F_{n}$. (We will write automorphisms on the right, although the topological representatives below will be written on the left).

We shall think of an outer automorphism $\Phi$ of $F_{n}$ as a coset, and as such it will be a set of automorphisms any two of which differ by conjugation by some element.

A similarity class in $\Phi$ will be an equivalence class under the equivalence relation, $\phi \sim \psi$ if and only if $\phi=\gamma_{g} \psi \gamma_{g^{-1}}$ for some $g \in F_{n}$. Note that we think of this as an equivalence relation on $\Phi$ where two equivalent automorphisms are called similar. 
It is important to note that, unlike the situation with matrices, two automorphisms are not called similar if they are conjugate. They are only similar if and only if they are conjugate by an inner automorphism. This follows the terminology of [7]. In [3], the same concept is denoted by the phrase conjugate up to inner automorphisms and some authors use instead the term isogredience.

The fixed subgroup of an automorphism $\phi \in \operatorname{Aut}\left(F_{n}\right)$ is the subgroup Fix $\phi=$ $\left\{w \in F_{n}: w \phi=w\right\}$ and by [2] has rank at most $n$. Given an outer automorphism $\Phi$, one can find (infinitely many) representatives $\phi_{i}$ of the distinct similarity classes in $\Phi$. It is clear that similar automorphisms have fixed subgroups of the same rank thus the following (possibly infinite) quantity is well defined.

Definition 2.1 The rank of an outer automorphism $\Phi$ of $F_{n}$ is the sum

$$
r(\Phi):=1+\sum \max \left(0, r\left(\operatorname{Fix} \phi_{i}\right)-1\right),
$$

where the sum is taken over a set $\left\{\phi_{i}\right\}$ of representatives, one for each similarity class of $\Phi$.

The following Theorem is proved in [8] and is in fact equivalent to the main Theorem of [2].

Theorem $2.2[8]$ For every $\Phi \in \operatorname{Out}\left(F_{n}\right), r(\Phi) \leq n$.

An immediate observation is that only finitely many of the $\phi_{i}$ have fixed subgroup of rank greater than one.

This observation leads us to the following definition.

Definition 2.3 For any $\Phi \in \operatorname{Out}\left(F_{n}\right)$, let $s(\Phi)$ denote the number of distinct similarity classes in $\Phi$ which have fixed subgroup of rank at least 2. By Theorem 2.2 , this quantity is always finite.

In [7] the index of an outer automorphism $i(\Phi)$ is defined. This has the same definition as the rank of $\Phi$ defined above with the addition of a term which counts attracting fixed points on the boundary of $F_{n}$. Thus it is clear that $r(\Phi) \leq i(\Phi)$. In [7] it is shown that $i(\Phi) \leq n$ for every $\Phi \in \operatorname{Out}\left(F_{n}\right) .{ }^{1}$ Also in [3] it is shown that if $\Phi$ is represented by a Dehn twist automorphism

\footnotetext{
${ }^{1}$ In fact the index described in [7] is one less than the one we refer to. The change is merely to emphasise the parallels between Theorems in $\operatorname{Out}\left(F_{n}\right)$ and $\operatorname{Aut}\left(F_{n}\right)$.
} 
(definitions below), then $i(\Phi)=n$ and $\Phi$ has no attracting points on the boundary. This is the same as saying that $r(\Phi)=n$. In this paper we prove the converse of this result. Namely that if $r(\Phi)=n$ then $\Phi$ can be represented by a Dehn twist automorphism.

In fact the main Theorem of [2], proved a conjecture of Scott's who formulated it after proving the following:

Theorem 2.4 [6] If an automorphism $\phi$ of $\operatorname{Aut}\left(F_{n}\right)$ has finite order, then Fix $\phi$ is a free factor of $F_{n}$.

Corollary 2.5 For any $\phi \in \operatorname{Aut}\left(F_{n}\right)$ and any integer $m \geq 1$, Fix $\phi$ is a free factor of Fix $\phi^{m}$.

Proof By Theorem 2.2, Fix $\phi^{m}$ is of finite rank and as $\phi$ and $\phi^{m}$ commute, $\phi$ leaves Fix $\phi^{m}$ invariant. Since it acts as a finite order automorphism, the result follows from Theorem 2.4.

This will have important consequences for us. The construction used in the proof of the following Proposition is due to G. Levitt.

Lemma 2.6 Consider an outer automorphism $\Phi \in$ Out $\left(F_{n}\right)$ of finite order. If $\Phi$ is not the identity then $r(\Phi)<n$.

Proof We may find a maximal set of automorphisms $\phi_{1}, \ldots, \phi_{k} \in \Phi$ in distinct similarity classes all of whose fixed subgroups have rank at least 2. (Note that $k=s(\Phi)$.) Thus $r(\Phi)=1+\sum_{j=1}^{k}\left(r\left(\operatorname{Fix} \phi_{j}\right)-1\right)$.

Also, we know that $\Phi$ has order $m$ in $\operatorname{Out}\left(F_{n}\right)$ for some $m \geq 2$. Hence every automorphism in $\Phi^{m}$ is inner and by Theorem 2.4 this implies that $\phi_{j}{ }^{m}=1 \in$ $\operatorname{Aut}\left(F_{n}\right)$ for all $1 \leq j \leq k$.

Pick a basis $x_{1}, \ldots, x_{n}$ for $F_{n}$ and find $g_{2}, \ldots, g_{k}$ so that $\phi_{j} \gamma_{g_{j}}=\phi_{1}$. (By definition the $\phi_{j}$ differ by inner automorphisms.)

Consider a free group of rank $n+k-1, F$, with basis $x_{1}, \ldots, x_{n}, \ldots, x_{n+k-1}$ where we identify $F_{n}$ with $\left\langle x_{1}, \ldots, x_{n}\right\rangle$. Define an automorphism $\phi$ of $F$ by setting $\left.\phi\right|_{F_{n}}=\phi_{1}$ and $\left(x_{n+j-1}\right) \phi=x_{n+j-1} g_{j}, 2 \leq j \leq k$.

First note that $\phi$ cannot fix certain words. $\phi$ cannot fix any word of the form $x_{j} w x_{j^{\prime}}{ }^{-1}$ for $j \neq j^{\prime} \geq n+1$ and $w \in F_{n}$, for if it did then this would imply that

$$
\begin{aligned}
g_{j}(w) \phi_{1} g_{j^{\prime}}-1 & =w \Rightarrow \\
w^{-1}(w) \phi_{j} & =g_{j^{\prime}} g_{j}^{-1}, \text { since } \phi_{j} \gamma_{g_{j}}=\phi_{1} .
\end{aligned}
$$


This last equality is not possible since it would mean that

$$
\begin{aligned}
\gamma_{w}{ }^{-1} \phi_{j} \gamma_{w} & =\phi_{j} \gamma_{\left(w^{-1}\right) \phi_{j} w} \\
& =\phi_{j} \gamma_{g_{j} g_{j^{\prime}}-1} \\
& =\phi_{j} \gamma_{g_{j}} \gamma_{g_{j^{\prime}}-1} \\
& =\phi_{1} \gamma_{g_{j^{\prime}}-1} \\
& =\phi_{j^{\prime}}
\end{aligned}
$$

and by construction these automorphisms are not similar.

Also, $\phi$ cannot fix any word of the form $x_{j} w$, for $j \geq n+1$ and $w \in F_{n}$ for then we get $g_{j}=w\left(w^{-1}\right) \phi$. This would imply the similarity of $\phi_{1}$ and $\phi_{j}$ as $\gamma_{w} \phi_{1} \gamma_{w}{ }^{-1}=\phi_{j}$ again reaching a contradiction.

We are now in a position to determine Fix $\phi$.

\section{Claim}

$$
\text { Fix } \phi=\text { Fix } \phi_{1} *_{j=2}^{k} x_{n+j-1}\left(\text { Fix } \phi_{j}\right) x_{n+j-1}{ }^{-1} .
$$

It is clear that the term on the right hand side is a subgroup of Fix $\phi$. Consider a word $w \notin F_{n}$, of shortest length fixed by $\phi$ and not of the form given above. We can write such a word as,

$$
w_{0} x_{j_{1}}{ }^{\epsilon_{1}} w_{1} x_{j_{2}}{ }^{\epsilon_{2}} w_{2} \ldots x_{j_{p}}{ }^{\epsilon_{p}} w_{p}
$$

where each $j_{i} \geq n+1, \epsilon_{i}= \pm 1$ and $w_{i} \in F_{n}$ and $p \geq 1$. We proceed by a simple cancellation argument.

If $\epsilon_{1}=-1$ it is easy to see that $x_{j_{1}} w_{0}{ }^{-1}$ must be fixed, giving a contradiction as above. Hence $\epsilon_{1}=1$ and since this implies that $w_{0}$ is fixed we may assume that $w_{0}=1$ by minimality of the length of $w$.

Now, if $\epsilon=1$ and either $p=1$ or $\epsilon_{2}=1$, then $x_{j_{1}} w_{1}$ must be fixed, again a contradiction. Thus $p$ must be at least $2, \epsilon_{1}=1$ and $\epsilon_{2}=-1$ leading us to the conclusion that $x_{j_{1}} w_{1} x_{j_{2}}{ }^{-1}$ is fixed. The only way that $\phi$ can fix a word of this type is if $j_{1}=j_{2}$ and $w_{1} \in$ Fix $\phi_{j_{1}}$. This contradicts the minimality of $w$ and proves the claim.

Hence,

$$
r(\operatorname{Fix} \phi)=\sum_{j=1}^{k} r\left(\text { Fix } \phi_{j}\right)=r(\Phi)+k-1 .
$$

However, each $\phi_{j}$ has order $m$ and since $\phi_{j}=\phi_{1} \gamma_{g_{j}-1}$, this implies that $\left(g_{j}^{-1}\right) \phi_{1}^{m-1}\left(g_{j}^{-1}\right) \phi_{1}^{m-2} \ldots g_{j}^{-1}=1$. 
Taking inverses we get that $g_{j}\left(g_{j}\right) \phi_{1}\left(g_{j}\right) \phi_{1}^{2} \ldots\left(g_{j}\right) \phi_{1}^{m-1}=1$ and hence that $x_{j} \phi^{m}=x_{j}$. Since $\left.\phi^{m}\right|_{F_{n}}=\phi_{1}^{m}=1$ we know that $\phi^{m}=1$. Clearly, $\phi \neq 1$ and so by Theorem 2.4, $r($ Fix $\phi)<r(F)=n+k-1$. Thus $r(\Phi)=r($ Fix $\phi)-k+1<$ $n$, completing the proof of the Lemma.

Consider an outer automorphism $\Phi$. Clearly if $\phi, \psi \in \Phi$ are similar, then $\phi^{m}, \psi^{m} \in \Phi^{m}$ will also be similar. The converse on the other hand need not be true. However, if we concentrate on those similarity classes with non-trivial fixed subgroup, the next proposition tells us that the only way these similarity classes can get 'collapsed' in a power is if the rank of the outer automorphism increases.

Proposition 2.7 Consider $\Phi \in \operatorname{Out}\left(F_{n}\right)$ and let $\phi, \psi \in \Phi$ be non-similar automorphisms each with non-trivial fixed subgroup. If for some integer $m$, $\phi^{m}$ and $\psi^{m}$ are similar, then $r(\Phi)<r\left(\Phi^{m}\right)$.

Proof We first choose a collection of automorphisms $\phi_{1}, \ldots, \phi_{k} \in \Phi$ in distinct similarity classes each with non-trivial subgroup so that each of $\phi$ and $\psi$ is similar to some automorphism on the list. Additionally, we enlarge the list so that any automorphism in $\Phi$ which has fixed subgroup of rank at least 2 is similar to one on the list.

After a rearrangement we may find integers $k_{1}, \ldots, k_{s}$ so that $\phi_{i}^{m}$ is similar to $\phi_{j}^{m}$ if and only if $k_{p} \leq i, j<k_{p+1}$ for some $1 \leq k_{p}<k_{s}$. In other words, we list representatives of similarity classes for $\Phi$ so that only consecutive similarity classes get collapsed in $\Phi^{m}$. As a consequence, the automorphisms $\phi_{k_{1}}^{m}, \phi_{k_{2}}^{m}, \ldots, \phi_{k_{s}}^{m}$ form a set of representatives of distinct similarity classes of $\Phi^{m}$ with non-trivial fixed subgroup. Thus

$$
r\left(\Phi^{m}\right) \geq 1+\sum_{p=1}^{s}\left(r\left(\operatorname{Fix} \phi_{k_{p}}^{m}\right)-1\right) .
$$

By changing representatives for similarity classes in $\Phi$ we may in fact assume that $\phi_{i}^{m}=\phi_{j}^{m}$ whenever $k_{p} \leq i, j<k_{p+1}$. Thus if $k_{p} \leq j<k_{p+1}$, then Fix $\phi_{j}$ is a subgroup of Fix $\phi_{j}^{m}=$ Fix $\phi_{k_{p}}^{m}:=H_{p}$. In fact, as $\phi_{j}$ and $\phi_{j}^{m}$ commute, $H_{p}$ is invariant under $\phi_{j}$ which restricts to a finite order automorphism on this subgroup.

Also, if we write $\phi_{j}=\phi_{k_{p}} \gamma_{g}$ then,

$$
\begin{aligned}
\phi_{j}^{m} & =\phi_{k_{p}}^{m} \gamma_{(g) \phi_{k_{p}}^{m-1}(g) \phi_{k_{p}}^{m-2} \ldots(g) \phi_{k_{p}} g} \\
& =\phi_{j}^{m} \gamma_{(g) \phi_{k_{p}}^{m-1}(g) \phi_{k_{p}}^{m-2} \ldots(g) \phi_{k_{p}} g}
\end{aligned}
$$

Algebraic 83 Geometric Topology, Volume 2 (2002) 
and hence $(g) \phi_{k_{p}}^{m-1}(g) \phi_{k_{p}}^{m-2} \ldots(g) \phi_{k_{p}} g=1$. Looking at the image of this element under $\phi_{k_{p}}$ we note that $(g) \phi_{k_{p}}^{m} g^{-1}=1$. In other words, $g \in \operatorname{Fix} \phi_{k_{p}}^{m}=H_{p}$ and the two automorphisms in question induce the same outer automorphism when restricted to $H_{p}$.

Note that if $g$ were to be fixed by $\phi_{k_{p}}$ then this would imply that that $g=1$ and so that $\phi_{j}=\phi_{k_{p}}$. Hence if $k_{p+1}-k_{p}>1$ then $H_{p}=$ Fix $\phi_{k_{p}}^{m}$ is strictly larger than Fix $\phi_{k_{p}}$ and in particular, the outer automorphism induced by $\phi_{k_{p}}$ on $H_{p}$ cannot be the identity.

Thus let $\Psi_{p}$ be the outer automorphism induced by the restriction of $\phi_{k_{p}}$ to $H_{p}$. By the comments above, we know that $\Psi_{p}$ is a finite order outer automorphism and that,

$$
r\left(\Psi_{p}\right) \geq 1+\sum_{j=k_{p}}^{k_{p+1}-1}\left(r\left(\operatorname{Fix} \phi_{j}\right)-1\right) .
$$

Note that $r\left(\Psi_{p}\right)$ is always bounded above by $r\left(H_{p}\right)$ by Theorem 2.2 , however if the number of terms in the sum on the right hand side is greater than one we know that $\Psi_{p} \neq 1$ and hence we may apply Lemma 2.6 to deduce that $r\left(\Psi_{p}\right)<r\left(H_{p}\right)$. In fact, our hypothesis guarantees that for some $p$ this will be the case, and so

$$
1+\sum_{p=1}^{s}\left(r\left(\Psi_{p}\right)-1\right)<1+\sum_{i=1}^{s}\left(r\left(H_{p}\right)-1\right) .
$$

As the left hand side is bounded below by $r(\Phi)$ and the right hand side is bounded above by $r\left(\Phi^{m}\right)$ this concludes the proof.

Recall that $w \in F_{n}$ is $\phi$ periodic if $(w) \phi^{m}=w$ for some $m \neq 0$.

Corollary 2.8 Let $\Phi \in \operatorname{Out}\left(F_{n}\right)$ have maximal rank and suppose that $w \in F_{n}$ is $\phi$ periodic for some $\phi \in \Phi$ with non-trivial fixed subgroup. Then $w \in$ Fix $\phi$.

Proof Choose an integer $m$ such that $w \phi^{m}=w$. Since $r(\Phi)=n$ we deduce, by Proposition 2.7, that if $\phi, \psi \in \Phi$ have non-trivial fixed subgroup, then they are similar if and only if $\phi^{m}, \psi^{m} \in \Phi^{m}$ are similar. In particular this means that if $r($ Fix $\phi)<r\left(\right.$ Fix $\left.\phi^{m}\right)$ then $r(\Phi)<r\left(\Phi^{m}\right)$, a contradiction. But by Corollary 2.5, if $r($ Fix $\phi)=r\left(\right.$ Fix $\left.\phi^{m}\right)$ then in fact Fix $\phi=$ Fix $\phi^{m}$. This completes the proof. 


\section{Relative train track maps}

We use the relative train track maps of Bestvina and Handel to analyze an outer automorphism. We recap some of the properties of relative train track maps.

A relative train track map is a self homotopy equivalence, $f$, of a graph $G$ which maps vertices to vertices and edges to paths. Such an $f$ is called a topological representative.

Note that if the image $f(e)$ of an edge $e$ is not homotopic to a trivial path relative endpoints then we may replace $f$ with a homotopically equivalent map that is locally injective on the interior of $e$. The process of doing this for each edge is called tightening $f$. Relative train track maps are always tightened.

The graph $G$ has no valence one vertices and is maximally filtered in the sense that it has subgraphs

$$
\emptyset=G_{0} \subseteq G_{1} \subseteq \cdots \subseteq G_{m}=G,
$$

where each $G_{i}$ is an $f$-invariant subgraph and if $f\left(G_{r}\right) \nsubseteq G_{r-1}$ then there is no $f$-invariant subgraph strictly between $G_{r-1}$ and $G_{r}$. The closure of $G_{r} \backslash G_{r-1}$ is denoted by $H_{r}$ and is called the $r^{\text {th }}$ stratum.

On labelling the edges of the $r^{\text {th }}$ stratum, $e_{1}, \ldots, e_{k}$, one can form the $r^{\text {th }}$ transition matrix, $M_{r}$, whose $(i, j)$ entry is the number of times that that $f\left(e_{i}\right)$ crosses $e_{j}$ (in either direction).

If $f\left(G_{r}\right) \subseteq G_{r-1}$ then $M_{r}$ is a zero matrix and $H_{r}$ is called a zero stratum. If $M_{r}$ is a permutation matrix, then $H_{r}$ is called a level stratum. Otherwise $H_{r}$ is called an exponential stratum.

Remark 3.1 In order for $f, G$ to be a relative train track map further conditions need to be imposed on the exponential strata. However, we shall only need to consider, by Proposition 4.1, those maps with no exponential strata, in which case relative train track maps are precisely those topological representatives which are tight and maximally filtered.

A Nielsen path (NP) is a path in $G$ which is fixed by $f$ up to homotopy relative endpoints. An indivisible Nielsen path (INP) is an NP which cannot be written (non-trivially) as the concatenation of NP's. In [2] it is shown that every NP can be written uniquely as a product of INP's.

A path in $G$ is said to have height $r$ if it is contained in $G_{r}$ but not $G_{r-1}$. In [2] it is shown that there is at most one INP of height $r$ for each $r$. Note that this 
uses the property known as stability in [2]. We shall also assume throughout that isolated fixed points of $f$ are in fact vertices of $G$. The following remarks are part of the analysis of [2] and in particular, the proof of Proposition 6.3.

Remark 3.2 ([2], pp48-49) If there is an INP, $\rho$, of height $r$, then $H_{r}$ cannot be a zero stratum. Furthermore, if $H_{r}$ is a level stratum then it must consist of a single edge $E$, with $f(E)=E u$ for some path $u$ in $G_{r-1}$. In that case, $\rho$ must be of the form $E \beta$ or $E \beta \bar{E}$ for some path $\beta$ in $G_{r-1}$.

A graph $\Sigma$ along with a map $p: \Sigma \rightarrow G$ may then be constructed such that,

(1) $p$ maps vertices of $\Sigma$ to vertices of $G$ fixed by $f$,

(2) $p$ maps edges of $\Sigma$ to INPs, and

(3) every NP in $G$ is the image (under $p$ ) of a path in $\Sigma$.

In fact, $\Sigma$ is constructed so that its vertices can be regarded as being precisely those vertices of $G$ which are fixed by $f$. Also, following [2], one may define certain subgraphs of $\Sigma$.

Definition 3.3 Let $\Sigma_{r}$ to be the (not necessarily connected) maximal subgraph of $\Sigma$ which maps to $G_{r}$ under $p$.

Definition 3.4 For every vertex $v$ of $G$, which is fixed by $f, \Sigma^{v}$ is the component of $\Sigma$ containing $v$.

Remark 3.5 ([2], p48) The graph $\Sigma_{r}$ differs from $\Sigma_{r-1}$ by at most a single edge when there is an INP of height $r$.

For a connected graph, $G$, define the rank of $G, r(G)$ to be the rank of $\pi_{1}(G)$. For an arbitrary graph define the reduced rank to be

$$
\widetilde{r}(G)=1+\sum \max \left(0, r\left(G_{k}\right)-1\right)
$$

where the sum ranges over the components of $G$.

It is shown in [2], p48, that

(1) $\widetilde{r}(\Sigma) \leq \widetilde{r}(G)=r(G)$ and,

(2) $\widetilde{r}\left(\Sigma_{r}\right) \leq \widetilde{r}\left(G_{r}\right)$.

By Remark 3.5 above we also have that

(3) $\tilde{r}\left(\Sigma_{r+1}\right) \leq \widetilde{r}\left(\Sigma_{r}\right)+1$.

Algebraic 83 Geometric Topology, Volume 2 (2002) 
Remark 3.6 ([2], p48) Note that if $\widetilde{r}\left(\Sigma_{r+1}\right)=\widetilde{r}\left(\Sigma_{r}\right)+1$ then there is an edge in $\Sigma$ (which maps to an INP, $\rho_{r}$ of height $r$ in $G$ ) and which has endpoints in (possibly one) non-contractible components of $\Sigma_{r}$. Thus $\rho_{r}$ also has endpoints in (possibly one) non-contractible components of $G_{r}$ and so $\widetilde{r}\left(G_{r+1}\right) \geq \widetilde{r}\left(G_{r}\right)+$ 1 .

\section{Representing automorphisms}

Let $f$ be a relative train track map on the graph $G$. Suppose that $v$ is a vertex of $G$ and $\mu$ a path in $G$ from $f(v)$ to $v$. Then $\pi_{1}(f, \mu)$ will denote the induced isomorphism of $\pi_{1}(G, v)$ that sends the closed path $\alpha$ at $v$ to $\bar{\mu} f(\alpha) \mu$. Here $\bar{\mu}$ denotes the inverse path to $\mu$. Write $\pi_{1}(f, v)$ in the case where $v$ is fixed by $f$ and $\mu$ is the trivial path at $v$.

Let $R_{n}$ denote the graph with one vertex, $*$, and $n$ edges, called the rose and identify $F_{n}$ with $\pi_{1}\left(R_{n}, *\right)$. We say that an outer automorphism $\Phi \in \operatorname{Out}\left(F_{n}\right)$ is represented by the relative train track map $f$ on $G$ if there is a homotopy equivalence, $\tau: R_{n} \rightarrow G$ such that the following diagram commutes up to free homotopy:

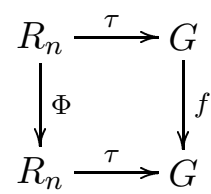

Note that we are identifying $\Phi$ with a self homotopy equivalence of $R_{n}$.

Given a representation of $\Phi$ as above, we say that $\phi \in \Phi$ is point represented at $v$ (by $f, G, \tau$ ) if $v$ is fixed by $f$ and there is a path, $\alpha$, from $\tau(*)$ to $v$ such that the following diagram commutes,

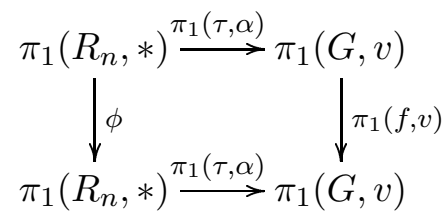

where $\pi_{1}(\tau, \alpha)$ is induced by the map which sends the path $g \subset R_{n}$ to $\bar{\alpha} \tau(g) \alpha$. It is shown in [2] that every outer automorphism, $\Phi$, is represented by a relative train track map, $f, G, \tau$. Furthermore we have:

Proposition 3.7 (Corollary 2.2, [2]) If an (ordinary) automorphism, $\phi \in \Phi$ has fixed subgroup of rank at least 2 , then this automorphism will be point represented by $(f, G, \tau)$. 
Note that if $\phi$ is point represented at $v$, then any automorphism similar to $\phi$ will also be point represented at $v$. Also, if there is a Nielsen path between the vertices $v$ and $v^{\prime}$, then $\phi$ will be point represented at $v^{\prime}$. Conversely, suppose that $\phi$ is point represented at both $v$ and $v^{\prime}$, with paths $\alpha, \alpha^{\prime}$ from $\tau(*)$ to $v, v^{\prime}$ respectively, then $\bar{\alpha} \alpha^{\prime}$ is a Nielsen path from $v$ to $v^{\prime}$.

Remark 3.8 Hence, bringing this together, if $\phi, \phi^{\prime} \in \Phi$ are both point represented at $v$ and $v^{\prime}$ respectively (by $f, G, \tau$ ) then they are similar if and only if there is a Nielsen path from $v$ to $v^{\prime}$. In the case where all NP's are closed, each fixed vertex will determine a distinct similarity class of $\Phi$.

If $\Phi$ is represented by $f, G, \tau$ and $\phi \in \Phi$ is point represented at $v$ then (Definition 3.4) $r\left(\Sigma^{v}\right)=r($ Fix $\phi)$ and $\widetilde{r}(\Sigma)=r(\Phi)$. In fact the map $p: \Sigma \rightarrow G$ induces isomorphisms from $\pi_{1}\left(\Sigma^{v}\right)$ to Fix $\phi$ whenever $\phi$ is point represented at $v$.

In the case where $\Phi$ has maximal rank (and is represented by $f, G, \tau$ ) then $\widetilde{r}(\Sigma)=r(G)$. By Remarks 3.5 and 3.6 we deduce that:

Lemma 3.9 If $\Phi$ has maximal rank then,

$$
\widetilde{r}\left(\Sigma_{k}\right)=\widetilde{r}\left(G_{k}\right) \text { for all } k .
$$

\section{Good Representatives}

From now on $\Phi$ will be a maximal rank outer automorphism of $F_{n}$. We shall show in this section that every such outer automorphism has a relative train track map representative with good properties. The first step is to observe:

Proposition 4.1 (Prop 4, [5]) If $\Phi$ has maximal rank then any relative train track map representative has no exponential strata.

In fact the Proposition in [5] relates to automorphisms (not outer!) of $F_{n}$ with fixed subgroup of rank $n$. However, the only hypothesis used is that $\widetilde{r}\left(\Sigma_{k}\right)=\widetilde{r}\left(G_{k}\right)$ for all $k$, and hence the proof there applies equally in our situation.

In order to find a good relative train track map representative, we need to perform a certain operation as follows. Suppose that $f: G \rightarrow G$ is a relative train track map and that $H_{k}=\{E\}$, where $f(E)=E u$, and $u$ is a path in 
$G_{k-1}$. For any path $\alpha$ in $G_{k-1}$ with initial vertex the same as the terminal vertex of $E$ we can define a new graph $G^{\prime}$ by replacing $E$ with an edge $E^{\prime}$ with the same initial vertex as $v$ and whose terminal vertex is the same as that of $\alpha$. Every edge of $G-\{E\}$ is naturally identified with that of $G^{\prime}-\left\{E^{\prime}\right\}$. We can then define $f^{\prime}: G^{\prime} \rightarrow G^{\prime}$ so as to agree with $f$ (up to homotopy) on $G-\{E\}$, and so that $f^{\prime}\left(E^{\prime}\right) \simeq f(E) \bar{\alpha} u f(\alpha)$ and $f^{\prime}$ is tight. The homotopy equivalence $p: G \rightarrow G^{\prime}$ which is the 'identity' on $G-\{E\}$ and sends $E$ to $E^{\prime} \bar{\alpha}$ gives the following commuting diagram.

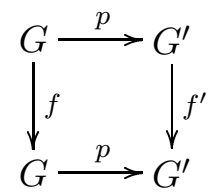

Moreover, if we set $G_{j}^{\prime}=p\left(G_{j}\right)$ then $f^{\prime}$ is a relative train track map with stratum $H_{j}^{\prime}$ of the same type (zero, level or exponential) as $H_{j}$. This operation is called sliding in [1] and a proof of the above statements is contained in [1], Lemma 5.4.1 and is a slight variation of the construction that appears in [5], Proposition 3.

Our first application of sliding is in fact precisely analogous to that in [5].

Proposition 4.2 Let $\Phi \in \operatorname{Out}\left(F_{n}\right), n \geq 2$, have maximal rank. Then there is a relative train track map representative, $f, G$, for $\Phi$ in which every indivisible Nielsen path, $\rho_{k}$, of height $k$ is either of the form $E \beta \bar{E}$ for some path $\beta$ in $G_{k-1}$ or $\rho_{k}=E$ and $E$ is a closed loop.

Proof By Proposition 4.1 and Remark 3.2, we only need to consider the case where a stratum $H_{k}$ consists of a single edge $E, f(E)=E u$, and $E \alpha$ is an INP, for some $u, \alpha$ subpaths in $G_{k-1}$. (This requires subdivision at isolated fixed points). Sliding $E$ along $\alpha$ we obtain a relative train track map representative $f^{\prime}, G^{\prime}$, where $f\left(E^{\prime}\right)=E^{\prime}$. If we do this in all possible cases and then collapse any fixed edges which are not loops, we end up deleting some strata, but otherwise still with a relative train track map representative. It is clear that for this map, every INP is of one of the above types.

An examination of the above proof gives a way of starting from a representative of $\Phi$ and getting another where we have better control of INP's. We want to have an easy way of insuring this condition. For that we need the following:

Definition 4.3 Let $f, G$ represent the maximal rank outer automorphism $\Phi$. We say that $f, G$ has minimal complexity if $G$ has the minimal number of 
vertices amongst all representatives of $\Phi$ subject to the restriction that $f, G$ satisfies the conclusion to Proposition 4.2 and that all isolated fixed points are vertices.

Lemma 4.4 Any representative $f, G$ of $\Phi$ of minimal complexity has the minimal number of vertices amongst all representatives of $\Phi$ with vertices at isolated fixed points.

Proof An examination of the proof to Proposition 4.2 shows that if a representative with vertices at isolated fixed points does not satisfy the proposition then we perform a sliding operation followed by the collapse of an invariant forest. Since this cannot increase the number of vertices of the underlying graph and cannot introduce any new isolated fixed points, we are done.

We shall henceforth assume that our maximal rank outer automorphism is represented by a relative train track map which satisfies the conclusions of Proposition 4.2.

Remark 4.5 Suppose that $G$ has exactly $r$ strata, so that $G=G_{r}$. Then since $G$ has no valence one vertices, $\widetilde{r} G_{r}>\widetilde{r} G_{r-1}$. Thus by Lemma 3.9, there is an INP of height $r$. Hence there is a single edge $E$ so that $H_{r}=\{E\}$ and $f(E)=E u$ with $u$ a path in $G_{r-1}$, possibly trivial. Denote the initial vertex of $E$ by $v$ and the terminal vertex by $w$. Let $C_{1}$ denote the component of $G_{r-1}$ containing $v$ and $C_{2}$ the component containing $w$. Thus if $E$ is nonseparating, $C_{1}=C_{2}$. Clearly, $f\left(C_{i}\right) \subseteq C_{i}$ and in fact it must restrict to a homotopy equivalence in each case.

Using this notation we can show:

Proposition 4.6 Let $f, G$ be a representative of $\Phi \in$ Out $\left(F_{n}\right)(n \geq 2)$ of minimal complexity. Then the following hold:

(1) If $E$ is separating in $G$ then $C_{2}$ has rank at least 2 .

(2) If $C_{1}$ is a rank 1 graph, then it consists of a single closed fixed edge and a single vertex.

Proof We start with property 1 where we need to show that if $E$ is separating then $C_{2}$ must have rank at least 2. If this is not the case then $C_{2}$ will have rank one and since there is an INP $E \beta \bar{E},\left.f\right|_{E \cup C_{2}}$ is homotopic to the identity map relative to $v$. Thus there is a map $f^{\prime}$ on $G$ which also represents $\Phi$ and 
which is the identity on $E \cup C_{2}\left(f^{\prime}\right.$ agrees with $f$ on $\left.C_{1}\right)$. It is clear that $f^{\prime}$ is also a relative train track map (with the same strata as $f$ ) and has vertices at isolated fixed points. Note that $E$ is a separating edge fixed by $f^{\prime}$. By collapsing $E$ we contradict Lemma 4.4.

To prove 2, note that since $\widetilde{r} \Sigma_{r}>\widetilde{r} \Sigma_{r-1}, \Sigma_{r-1}^{v}$ must have rank at least 1 . Hence there is always a closed Nielsen path at the vertex $v$ contained in $C_{1}$. So if the rank of $C_{1}$ is 1 then we can apply the argument as above to show that, without loss of generality, $f$ restricts to the identity on $C_{1}$. The only way that this does not contradict the minimal complexity hypothesis is if $C_{1}$ consists of a single fixed edge.

As an immediate corollary we get:

Corollary 4.7 Let $\Phi \in$ Out $\left(F_{2}\right)$ have maximal rank and $f, G$ be any relative train track map representative of minimal complexity. Then exactly one vertex $v$ and two edges, $a, b$, where without loss of generality $f(a) \simeq a$ and $f(b) \simeq b a^{r}$ for some integer $r$.

Proposition 4.8 Let $f, G$ be a relative train track map representing a maximal rank outer automorphism. Suppose that $C$ is a component of some $G_{k}$ with $r(C) \geq 1$. Then $f(C) \subseteq C$ and $f$ induces a maximal rank outer automorphism on $C$.

Proof The proof is by induction on $r(G)$. If $r(G)=1$ then $C$ can only be equal to $G$ and we are done.

Consider the edge $E$ as in Remark 4.5. If $E$ is a separating edge, then since it is the content of the highest stratum, we can write $\Sigma_{r-1}$ as a disjoint union of graphs, $\Sigma^{1}$ and $\Sigma^{2}$, where $p\left(\Sigma^{i}\right) \subseteq C_{i}$. In other words, $\Sigma^{i}$ contains all the edges of $\Sigma_{r-1}$ that map to INP's of $C_{i}$. It is clear by the properties of $\Sigma$ that the rank of the outer automorphism induced by $\left.f\right|_{C_{i}}$ is exactly $\widetilde{r} \Sigma^{i}$ and hence by Theorem 2.2, $\widetilde{r} \Sigma^{i} \leq \widetilde{r} C_{i}$. However,

$$
\begin{aligned}
\widetilde{r} G & =\widetilde{r} C_{1}+\widetilde{r} C_{2}+1 \\
& \geq \widetilde{r} \Sigma^{1}+\widetilde{r} \Sigma^{2}+1 \\
& =\widetilde{r} \Sigma_{r-1}+1 \\
& =\widetilde{r} \Sigma \\
& =\widetilde{r} G .
\end{aligned}
$$

The upshot of this is that each $\left.f\right|_{C_{i}}$ induces a maximal rank outer automorphism on $\pi_{1}\left(C_{i}\right)$. A similar argument applies when $E$ is non-separating, where 
$G-\{E\}=G_{r-1}=C_{1}=C_{2}$ to get that $\left.f\right|_{C_{1}}$ induces a maximal rank outer automorphism.

Now each $C_{i}$ inherits a filtration from $G$, namely, $C_{i} \cup G_{m}$ is an invariant subgraph of $C_{i}$. Thus as any component $C$ of some $G_{k}$ is actually a component of $C_{i} \cap G_{k}$ (except $G$ itself) we may use our inductive hypothesis to finish the proof.

Theorem 4.9 Let $f, G$ be a relative train track map of minimal complexity, with $r(G) \geq 2$, representing a maximal rank outer automorphism. Suppose that for some closed path $\alpha, f(\alpha) \simeq \bar{\mu} \alpha \mu$. Then there is a path $\eta$ in $G$ such that

(1) $[\bar{\eta} \alpha \eta] \in \pi_{1}(G, v)$.

(2) $\pi_{1}(f, v)$ has fixed subgroup of rank at least 2.

Proof Let $G=G_{r}$ and use the notation of Remark 4.5. Note that whether or not $E$ is separating, by Proposition $4.2, \Sigma$ differs from $\Sigma_{r-1}$ by a single closed loop at the vertex $v$. Since $\widetilde{r} \Sigma_{r}>\widetilde{r} \Sigma_{r-1}$, we must have that $r\left(\Sigma_{r-1}^{v}\right) \geq 1$ and $r\left(\Sigma^{v}\right) \geq 2$.

We will first prove the Theorem in the case where $\alpha$ is not freely homotopic to a path in $G_{r-1}$. By possibly replacing $\alpha$ with its inverse, we may choose an $\eta$ so that $\bar{\eta} \alpha \eta$ is a path that starts with $E$ and does not end with $\bar{E}$. Since this is a closed path at $v$ and $r\left(\Sigma^{v}\right) \geq 2$, it will suffice to show that this path is fixed up to homotopy.

Notice that in fact every positive $f$ iterate of this path also begins with $E$ and does not end with $\bar{E}$. Our key observation here is that, with respect to some basis, this path and its iterates are cyclically reduced words. If $E$ is nonseparating then choose any maximal tree that does not include $E$. The induced basis certainly ensures that each $\left[f^{k}(\bar{\eta} \alpha \eta)\right]$ are cyclically reduced.

If, on the other hand, $E$ is separating then note that $f$ induces automorphisms of $H=\pi_{1}\left(C_{1}, v\right)$ and on $K=\pi_{1}\left(C_{2} \cup\{E\}, v\right)$. If we choose a basis for $\pi_{1}(G, v)$ that extends bases for $H$ and $K$ it is then easy to see that $[\bar{\eta} \alpha \eta]$ starts with a non-trivial word from $K$ and ends with a non-trivial word from $H$ and the same will be true of all its iterates. Hence in either case $[\bar{\eta} \alpha \eta]$ and all its iterates are cyclically reduced elements of $\pi_{1}(G, v)$. But since any element of a free group has only finitely cyclically reduced conjugates, this means that $[\bar{\eta} \alpha \eta]$ is $\pi_{1}(f, v)$ periodic and hence by Corollary 2.8, fixed. 
This leaves us with the case where $\alpha$ is freely homotopic to a path in $G_{r-1}=$ $C_{1} \cup C_{2}$. Consider first the case where $\alpha$ is freely homotopic to a path in $C_{i}$ and $r\left(C_{i}\right)=1$. By Proposition 4.6, $C_{i}=C_{1}$ which consists of a fixed edge loop. Thus we may choose $\eta$ so that $\bar{\eta} \alpha \eta$ is fact a power of the fixed edge loop and we are done in this case.

If on the other hand $\alpha \subseteq C_{2}$ then $E$ must be a separating edge and the INP of height $r$ is of the form $E \beta \bar{E}$. We can then choose $\eta$ so that $\bar{\eta} \alpha \eta$ is a loop at $v$ homotopic to a power of that INP. (Recall we are assuming that $r\left(C_{2}\right)=1$.) Again we would be done.

We finish the argument by induction on $r(G)$. If $r(G)=2$ then either $\alpha$ is not freely homotopic to a path in $G_{r-1}$ or $\alpha \subseteq C_{i}$ where $r\left(C_{i}\right)=1$. The arguments above deal with each situation.

So suppose that the proposition is true for all rank less than $r(G)$ and at least 2. Again, if $\alpha$ is not freely homotopic to a path in $G_{r-1}$ we are done. Hence, without loss of generality $\alpha \subseteq C_{i}$ and we can assume that $r\left(C_{i}\right) \geq 2$. By Proposition 4.8 we may apply our induction hypothesis to complete the proof.

As an immediate consequence of the above we get:

Corollary 4.10 Let $\Phi \in \operatorname{Out}\left(F_{n}\right), n \geq 2$, be a maximal rank outer automorphism fixing a conjugacy class. Then there is a $\phi \in \Phi$ with fixed subgroup of rank at least 2 fixing an element of that conjugacy class.

We are now ready to show that a maximal rank outer automorphism of $F_{n}$ has a representative with very good properties, analagous to those in [5].

Theorem 4.11 Let $\Phi$ be a maximal rank outer automorphism of $F_{n}$. Then there is a relative train track map representative of minimal complexity for $\Phi$, $(f, G)$, such that,

(1) every vertex of $G$ is fixed,

(2) for every vertex, $v$ of $G, \pi_{1}(f, v)$ has fixed subgroup of rank at least 2 , and

(3) (up to orientation) every edge $E$ of $G$ satisfies, $f(E)=E \beta$, where $\beta$ is a closed Nielsen path contained in strata lower than $E$. 
Proof The case $n=2$ follows from Corollary 4.7, so we proceed by induction. Our hypothesis will actually be that given any representative of $\Phi$ of minimal complexity, a sequence of sliding operations will transform the representative into one which satisfies the conclusion of the Theorem.

Start with a relative train track map representative $f, G$ of minimal complexity and top edge $E$ as in Remark 4.5 so that the initial vertex of $E$ is $v$ and the terminal vertex is $w$. We already know that the fixed subgroup of $\pi_{1}(f, v)$ has rank at least 2 and that $v$ is a fixed vertex. In fact, we make make the following claim:

Claim After a sliding operation we obtain a relative train track map representative of minimal complexity with the following properties (notation from Remark 4.5):

(1) Either (i) $E$ is a fixed edge loop, or (ii) the INP of height $r$ is $E \beta \bar{E}$, where $\beta$ is a closed Nielsen path at $w$.

(2) In case (ii), $f(E)=E \beta^{k}$ for some $k$.

(3) $w$ is a fixed vertex and the fixed subgroup of $\pi_{1}(f, w)$ has rank at least 2 .

Proof of claim The claim is immediate if $E$ is a fixed edge loop. It also follows immediately from Proposition 4.6 if $E$ is non-separating and $r\left(C_{1}\right)=1$. Therefore (by another application of 4.6) we may assume that the INP of height $r$ is $E \beta \bar{E}$. So $\beta$ is a loop at $w$ where $\beta \subseteq C_{i}$ with $r\left(C_{i}\right) \geq 2$. Also (as in Remark 4.5), $f(E)=E u$ where $u$ is a path in $C_{i}$ (the same component of $G_{r-1}$ as $\beta$ ). By Proposition 4.8, there exists a path $\eta \subseteq C_{i} \subseteq G_{r-1}$ such that $\bar{\eta} \beta \eta$ is a closed Nielsen path at some vertex $w^{\prime}$ and that $\pi_{1}\left(f^{\prime}, w^{\prime}\right)$ has fixed subgroup of rank at least 2 . If we now slide $E$ along $\eta$, we get a new representative with an edge $E^{\prime}$, such that $f^{\prime}\left(E^{\prime}\right)=E^{\prime}[\bar{\eta} u f(\eta)]$. The new INP of height $r$ will be $E^{\prime}[\bar{\eta} \beta \eta] \bar{E}^{\prime}$.

However,

$$
f(\beta) \simeq \bar{u} \beta u
$$

and

$$
f(\bar{\eta} \beta \eta) \simeq \bar{\eta} \beta \eta .
$$

Hence $\bar{\eta} u f(\eta)$ commutes with $\bar{\eta} \beta \eta$ and since these are both closed paths we deduce that the former is a power of the latter. (Note $\beta$ cannot be a proper power.) Hence, $f^{\prime}\left(E^{\prime}\right)=E^{\prime} \beta^{\prime k}$ where $\beta^{\prime}$ is a closed Nielsen path at the vertex $w^{\prime}$. Moreover $w^{\prime}$ is fixed by $f^{\prime}, \pi_{1}\left(f^{\prime}, w^{\prime}\right)$ has fixed subgroup of rank at least 2 . 
Thus $f^{\prime}$ is a map with the required properties and since no new vertices were introduced, we may assume that the new representative has minimal complexity. This concludes the proof of the claim.

The idea now is to use the induction hypothesis on $C_{1}$ and $C_{2}$. (Sliding an edge in $C_{i}$ is equivalent to sliding the same edge in $G$ ). However it may be that $C_{1}, C_{2}$ contain valence one vertices (these are not valence one in $G$ but in the $C_{i}$ ) so we need to consider how this can arise. We proceed with a representative of $\Phi$ which satisfies the conditions in the claim above.

Consider first the case where $E$ is separating. Note that since the INP of height $r$ is a closed path at $v$, this implies that $\Sigma^{w}=\Sigma_{r-1}^{w}$ and hence that the fixed subgroup of $\pi_{1}(f, w)$ is contained in $\pi_{1}\left(C_{2}, w\right)$. Since distinct INP's start with distinct edges, we know that $w$ has valence at least 2 in $C_{2}$. Hence $C_{2}$ has no valence 1 vertices and applying the induction hypothesis we can assume that the Theorem holds for every edge and vertex of $C_{2}$. (Note here that $\left.f\right|_{C_{2}}$ is a relative train track map and if it were not of minimal complexity we could replace $\left.f\right|_{C_{2}}, C_{2}$ with some $f^{\prime}, C^{\prime}$ via a homotopy relative $w$. This is clearly not possible since it would contradict the minimal complexity of $f, G$ ).

To continue, if $r\left(C_{1}\right)=1$ we are done. Also, if every vertex of $C_{1}$ has valence at least 2 , then we are done since again we could apply the induction hypothesis to $\left.f\right|_{C_{1}}, C_{1}$.

So there is only something to prove if $r\left(C_{1}\right) \geq 2$ and $C_{1}$ has a valence one vertex. Clearly, $v$ is the only vertex of $C_{1}$ which can have valence 1 . Let $e$ be the edge of $C_{1}$ whose initial vertex is $v$. Since there is a closed INP at the vertex $v$ contained in $C_{1}$, we deduce that the INP is of the form $e \alpha \bar{e}$. Just as in the proof of the claim above, after sliding $e$, we can assume that $f(e)=e \alpha^{m}$ for some $m$ and that $\alpha$ is a closed Nielsen path. Note that the terminal vertex of $e$ must have valence at least 3, since otherwise we could slide $e$ along an edge to produce a valence 1 vertex in $G$, contradicting the property of minimal complexity. (This would also follow from the proof of the claim.) Also, both endpoints of $e$ are fixed and that the corresponding fixed subgroups have rank at least 2 in $\pi_{1}(G)$.

Hence $f(C-\{e\}) \subseteq C-\{e\}$ and every vertex has valence at least 2 . Thus we may apply our induction hypothesis to $C-\{e\}$. The Theorem is then is then proved in this case, since every edge of $G$ is either $E$, $e$ or in $C_{1} \cup C_{2}$, and every vertex is incident to one of these.

The same argument will apply when $E$ is non-separating, since we may assume that $r\left(C_{1}\right) \geq 2$ as the case $n=2$ has already been dealt with. 
Let us call a representative of $\Phi$ which satisfies the conclusions to Theorem 4.11 a good representative. One immediate observation is that since every NP is closed, by Remark 3.8, the number of vertices of a good representative is precisely $s(\Phi)$, the number of similarity classes with fixed subgroup at least 2 . Thus if we start with an automorphism $\phi \in \operatorname{Aut}\left(F_{n}\right)$ which has fixed subgroup of rank $n$, then a good representative of the outer automorphism induced by $\phi$ will have exactly one vertex. Moreover, $\phi$ will be point represented at that vertex and we recover the main Theorem of [5]. Methods used to analyze such automorphisms naturally generalise to our situation. Hence the argument used in [9] to solve the conjugacy problem for automorphisms with maximal fixed subgroup can be applied with almost no changes to get:

Theorem 4.12 Given two outer automorphisms of maximal rank, $\Phi, \Psi \in$ $\operatorname{Out}\left(F_{n}\right)$ in terms of images of a basis it is possible to decide whether they are conjugate.

We shall show below, that any outer automorphism of maximal rank can in fact be represented by a Dehn twist and the conjugacy problem has been solved for these in [3]. Thus the only advance made is an explicit algorithm when the automorphisms are given in terms of images on a basis.

\section{Graphs of groups and Dehn twists}

We shall now show that any outer automorphism of $F_{n}$ of maximal rank is represented by a Dehn Twist. We give a brief recap of the objects involved, taken from [3].

Definition 5.1 A graph of groups is given by

$$
\mathcal{G}=\left\{\Gamma(\mathcal{G}),\left\{G_{v}\right\}_{e \in E(\mathcal{G})},\left\{f_{e}\right\}_{e \in E(\mathcal{G})}\right\}
$$

$\Gamma(\mathcal{G})$ is a finite connected graph,

$V(\mathcal{G})$ is the vertex set of $\Gamma(\mathcal{G})$,

$E(\mathcal{G})$ is the (oriented) edge set of $\Gamma(\mathcal{G})$,

$G_{v}$ is the vertex group at $v \in V(\mathcal{G})$,

$G_{e}$ is the edge group at $e \in E(\mathcal{G})$ and,

$m_{e}: G_{e} \rightarrow G_{\tau(e)}$ is a monomorphism. (Here $\tau(e)$ denotes the terminal vertex of $e$ and hence $\tau \bar{e}$ the initial vertex.) 
The path group $\Pi(\mathcal{G})$ is the free product of the free group on the set $\left\{t_{e}: e \in\right.$ $E(\mathcal{G})\}$ with the groups $G_{v}$, subject to the relations,

(i) $t_{\bar{e}}=t_{e}^{-1}$ and

(ii) $t_{e} m_{e}(a) t_{e}{ }^{-1}=m_{\bar{e}}(a) \in G_{\tau \bar{e}}$ for all $a \in G_{e}, e \in E(\mathcal{G})$.

Every element of $\Pi(\mathcal{G})$ is given by a word

$$
W=r_{0} t_{1} \ldots t_{q} r_{q},
$$

where each $t_{i}=t_{e_{i}}$ and $r_{i}$ is an element of the free product of the $G_{v}$. Such a word is called a loop at the vertex $v$ if $r_{0}, r_{q} \in G_{v}, \tau\left(\overline{e_{1}}\right)=\tau e_{q}=v$ and $\tau e_{i}=\tau e_{i+1}^{-}$with $r_{i} \in G_{\tau\left(e_{i}\right)}$ for all $i$ (taking subscripts modulo $q$ ).

The set of loops at $v$ forms a subgroup of $\Pi(\mathcal{G})$ denoted, $\pi_{1}(\mathcal{G}, v)$ and called the fundamental group of $\mathcal{G}$ at $v$.

A Dehn twist, $\mathcal{D}$ on $\mathcal{G}$, with twistors $z_{e}$ is an automorphism of $\Pi(\mathcal{G})$ such that,

$$
\mathcal{D}\left(t_{e}\right)=t_{e} m_{e}\left(z_{e}\right)
$$

where $z_{e}$ is in the centre of $G_{e}$ and $z_{\bar{e}}=z_{e}{ }^{-1}$. Extend $D$ to the whole of $\Pi(\mathcal{G})$ by setting it equal to the identity on each vertex group. Note that specifying the twistors $z_{e}$ is sufficient to define $D$.

Since $D$ preserves incidence relations, it restricts to an automorphism $D_{v}$ on $\pi_{1}(\mathcal{G})$ for any $v \in V(\mathcal{G})$.

We shall say that an outer automorphism $\Phi \in O u t\left(F_{n}\right)$ is represented by a Dehn twist, $D$ on $\mathcal{G}$, at the vertex $v$, if there is an isomorphism $\sigma: F_{n} \rightarrow \pi_{1}(\mathcal{G}, v)$ and a $\phi \in \Phi$ such that the following diagram commutes,

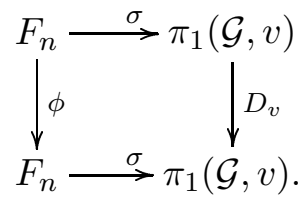

Note that $\pi_{1}(\mathcal{G}, v)$ and $\pi_{1}(\mathcal{G}, w)$ are conjugate in $\Pi(\mathcal{G})$ and that under this ismorphism, $D_{v}$ and $D_{w}$ define the same outer automorphism. Hence we may refer to the outer automorphism induced by $D$. In particular, if $\Phi$ is represented at the vertex $v$, then it will also be represented at every other vertex, with different choices of $\phi \in \Phi$.

Now it is clear that if $\Phi$ is represented by a Dehn twist $D$, then $\Phi$ and $D$ will have the same index, in fact they will also have the same rank (as outer automorphisms). In [3], Corollary 7.7 it is shown that every Dehn twist has 
maximal index and no attracting fixed infinite words. Since this is our definition of maximal rank, it follows that if $\Phi$ is represented by a Dehn Twist then it has maximal rank. We now prove the converse.

Theorem 5.2 Let $\Phi \in$ Out $\left(F_{n}\right), n \geq 2$ have maximal rank. Then $\Phi$ is represented by a Dehn twist.

Proof Start with a relative train track map representative, $f, G$, of $\Phi$ of minimal complexity satisfying the conclusion to Theorem 4.11. Form a graph $X$ from $G$ by deleting all fixed edge loops. Thus $X$ is a connected subgraph of $G$ on the same vertex set as $G$.

We now define a graph of groups $\mathcal{G}$ with graph $\Gamma(\mathcal{G})=X$. For each vertex $v$, let $G_{v}=$ Fix $\pi_{1}(f, v)$ and let each edge group $G_{e}$ be infinite cyclic with generator $a_{e}$. Now each edge, $e$, of $\Gamma(\mathcal{G})=X$ is also an edge of $G$ so we can determine the path $f(e)$ in $G$. By definition of $X, e$ is not a fixed edge loop and so, by Proposition 4.2 and Theorem 4.11, up to orientation there is an INP $e \beta_{e} \bar{e}$ and $f(e)=e \beta_{e}^{r_{e}}$ for some integer $r_{e}$ and a $\beta_{e}$ which is a closed INP at the endpoint $\tau(e)$ of $e$. We can then define a monomorphism $m_{e}: G_{e} \rightarrow G_{\tau(e)}$ by mapping $a_{e}$ to $\beta_{e}$. Similarly, $f_{\bar{e}}: G_{\bar{e}}=G_{e} \rightarrow G_{\tau \bar{e}}$ will map $a_{e}$ to $e \beta_{e} \bar{e}$. This completes the definition for $\mathcal{G}$.

Given an edge $e$ as above, set the twistor $z_{e}=a_{e}{ }^{r_{e}}$. (Recall that $f(e)=e \beta_{e}{ }^{r_{e}}$.) Since $G_{e}$ is abelian this clearly lies in the centre. Then with $\bar{z}_{e}=a_{e}{ }^{-r_{e}}$ we let $\mathcal{D}$ be the Dehn twist based on these twistors.

It should be noted that if $e$ has height $k$ in $G$, with respect to the stratification then there is a unique INP of height $k$ and since $f, G$ has minimal complexity this INP will be closed and of the form given by Proposition 4.2. Thus the definition of the maps $m_{e}$ for the graph of groups and the Dehn twist, $\mathcal{D}$ is unambiguous.

Now let $\Pi(G)$ denote the free group on the (unoriented) edge set of $G$ (so $e$ and $\bar{e}$ are considered inverse). Let $\sigma$ map an edge $e$ of $G$ to the corresponding stable letter $t_{e}$ of $\Pi(\mathcal{G})$ if $e$ is not a fixed edge loop. If $e$ is a fixed edge loop at $v$, let $\sigma(e)$ be the corresponding element of $G_{v}=$ Fix $\pi_{1}(f, v)$. Since $\Pi(G)$ is free, $\sigma$ extends to a unique homomorphism from $\Pi(G)$ to $\Pi(\mathcal{G})$. We claim that this is an isomorphism.

One can easily define the inverse, $\sigma^{\prime}$ from $\Pi(\mathcal{G})$ to $\Pi(G)$. Simply let $\sigma^{\prime}\left(t_{e}\right)=e$ for every stable letter $t_{e}$ and for each $r \in G_{v}=\operatorname{Fix} \pi_{1}(f, v)$, let $\sigma^{\prime}(r)$ be the unique product of INP's representing it. We can extend $\sigma^{\prime}$ to a well defined 
homomorphism after an easy check to show that the relations in $\Pi(\mathcal{G})$ are in the kernel of $\sigma^{\prime}$. It is then a matter of computation to show that the composition (in either order) of $\sigma$ and $\sigma^{\prime}$ is the identity, by just checking on generating sets. Thus $\sigma$ is an isomorphism as claimed.

Now as $f$ maps edges to edge paths, it induces an endomorphism $f_{*}$ on $\Pi(G)$. One can easily verify that the following diagram commutes,

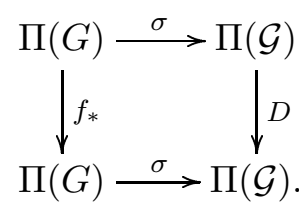

The map $D$ is the Dehn twist defined above. Note that $f_{*}$ is actually an automorphism of $\Pi(G)$ and one can prove this either by induction on the number of strata in $G$ or by observing the above diagram.

With this point of view, $\pi_{1}(G, v)$ is actually a subgroup of $\Pi(G)$ and $\pi_{1}(f, v)$ is the restriction of $f *$ to this subgroup. Since $\sigma$ preserves incidence relations one can immediately deduce that $\sigma$ restricts to an isomorphism between $\pi_{1}(G, v)$ and $\pi_{1}(\mathcal{G}, v)$ and thus the above commuting diagram restricts to,

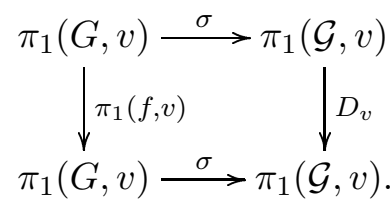

This concludes the proof of the Theorem.

Note that in our proof we have done more than show a maximal rank outer automorphism a represented by a Dehn twist. We have represented the outer automorphism when considered as a groupoid automorphism by a naturally equivalent Dehn twist, also considered as a groupoid automorphism. This highlights the strong connection between the two structures.

\section{Acknowledgements}

This work was completed with the support of an EPSRC fellowship.

Algebraic 83 Geometric Topology, Volume 2 (2002) 


\section{References}

[1] M. Bestvina and M. Feighn and M. Handel, The Tits Alternative for $\operatorname{Out}\left(F_{n}\right)$ I: Dynamics of Exponentially Growing Automorphisms. Annals of Math. 151 (2000), 517-623.

[2] M. Bestvina and M. Handel, Train tracks and automorphisms of free groups. Ann. of Math. (2) 135 (1992), 1-51.

[3] M. M. Cohen and M. Lustig, The conjugacy problem for Dehn twist automorphisms of free groups. Comment Math. Helv. 74 (1999) no. 2 179-200

[4] D. J. Collins and E. Turner, An automorphism of a free group of finite rank with maximal rank fixed point subgroup fixes a primitive element. J. Pure and Applied Algebra 88 (1993) 43-49

[5] D. J. Collins and E. Turner, All automorphisms of free groups with maximal rank fixed subgroups. Math. Proc. Cambridge Phil. Soc. 119 (1996), no. 4, 615630

[6] J. L. Dyer and G. P. Scott, Periodic automorphisms of free groups. Comm. Alg. 3 (1975), 195-201

[7] D. Gaboriau and A. Jaeger and G. Levitt and M. Lustig, An index for counting fixed points for automorphisms of free groups. Duke Math. J. 93 (1998), no. 3, $425-452$.

[8] D. Gaboriau and G. Levitt and M. Lustig, A dendrological proof of the Scott conjecture for automorphisms of free groups. Proc. Edinburgh Math. Soc. 41 (1998), no. 2, 325-322

[9] A. Martino, Normal forms for automorphisms of maximal rank. Quart. J. Math. 51 (2000), no. 4, 509-522

Department of Mathematics, University College Cork

Cork, Ireland

Email: A.Martino@ucc.ie

Received: 4 February 2002

Algebraic $6 \mathcal{G}$ Geometric $\mathcal{T}$ opology, Volume 2 (2002) 